Análisis y Modificación de Conducta, 2006, Vol. 32, No 143

\title{
LA RELACIÓN ENTRE LA FALTA DE SUEÑO, EL OCIO SE- DENTARIO Y EL SOBREPESO INFANTIL
}

\author{
Raquel Busto Zaplco \\ Isaac Amlgo Vázquez \\ Javier Herrero Díez \\ Concepción Fernández Rodríguez
}

Universidad de Oviedo

\section{RESUMEN}

En esta investigación se estudio, a través del path analysis, la relación entre los hábitos de ocio sedentario, sueño y el ĺndice de Masa Corporal (IMC). Participó una muestra de 72 alumnos, 41 chicas y 31 chicos, de cuarto de primaria con una edad comprendida entre los 9 y los 10 años y medio. Para ello se realizó una entrevista individual en la que se preguntaba a los niños los programas que veían cada uno de los días de la semana y los juegos con los que jugaban con la consola y el ordenador. Los resultados pusieron de manifiesto que los niños que duermen menos emplean más tiempo realizando alguna forma de ocio sedentario y que esto se relaciona a su vez con un IMC más elevado. Se discuten las implicaciones sobre el estudio del estilo de vida y sobrepeso infantil.

Palabras clave: FALTA DE SUEÑO, OCIO SEDENTARIO Y SOBREPESO INFANTL. 


\section{SUMMARY}

The objective of this research was to analyse the relations between short sleeping, sedentary leisure and childhood overweight using the path analysis. The sample consisted of 72 participants aged 9 to 10 and half years, 41 girls and 31 boys. One individual interview was used in order to evaluate these variables. The results pointed out that participants with short sleeping spent more time watching TV and playing videogames o using computer and in the same way this type of leisure is related with a higher Body Mass Index. The implications on the study of childhood lifestyle and overweight are discussed.

\section{Key words: SHORT SLEEPING, SEDENTARY LEISURE, CHILDHOOD} OVERWEIGHT.

Existe un amplio consenso entre los investigadores sobre el hecho de que la obesidad infantil puede llegar a convertirse en una pandemia a lo largo de este siglo. La realidad es que el incremento del sobrepeso y la obesidad infantil, que parece tener una relación muy estrecha con el estilo de vida de las sociedades desarrolladas, ha hecho sonar con fuerza las alarmas del sistema sanitario en los últimos años, tras registrarse un crecimiento espectacular de este problema en relación a las últimas décadas del siglo pasado. Particularmente, la obesidad tanto infantil como adulta ha mostrado una curva de crecimiento similar tanto en niños como adultos desde mediados de los años ochenta (Flegal, 2005). En España se ha pasado de un porcentaje de niños obesos del $5 \%$ en 1990 hasta el $16 \%$ en la actualidad. Eso significa que el crecimiento de la obesidad infantil está alrededor del $0.5 \%$ anual. Este veloz crecimiento de los problemas de exceso de peso elimina la posibilidad de cualquier explicación del mismo en términos genéticos. Tan sólo aproximadamente un $7 \%$ de los casos de sobrepeso u obesidad tienen una causa biológica mientras que el $93 \%$ restante corresponde a una obesidad exógena, es decir, a aquélla que tiene su causa en un saldo positivo entre el consumo de calorías y el gasto energético del organismo. 
No es de extrañar, entonces, que el crecimiento de la obesidad siga siendo vertiginoso: mientras que en 1998 tan sólo el $7 \%$ de los Estados de Norteamérica tenían una prevalencia de obesidad infantil superior al $20 \%$ de la población, en 2000 ya eran el $50 \%$ de los Estados los que alcanzaban esa cifra, y en 2002 tres Estados norteamericanos superaban entre su población un porcentaje de obesidad infantil del $25 \%$. Las curvas de crecimiento de la obesidad infantil en España durante los últimos años y las que se dieron en los Estados Unidos hace dos décadas escasas son tan llamativamente parecidas que todo indica que la situación sanitaria norteamericana actual vinculada al exceso de peso infantil va a darse con toda probabilidad en nuestro país dentro de algunos años.

Las complicaciones de salud derivadas de la obesidad son múltiples, tanto en la infancia como en la edad adulta. En su informe de 2002, la Organización Mundial de la Salud señalaba que la obesidad está ligada al $60 \%$ de las muertes producidas por enfermedades no contagiosas, principalmente cáncer, trastornos cardiovasculares y diabetes (Garcés, Gutierrez y Benavenente, 2005). Por otro lado, la obesidad infantil y la obesidad adulta se encuentran relacionadas, en la medida en que casi ocho de cada diez niños obesos terminan convirtiéndose en adultos obesos. Los problemas médicos derivados de la obesidad infantil son numerosos. Por nombrar únicamente los más llamativos, cabe destacar que han comenzado a detectarse casos sorprendentes de diabetes tipo II no insulino-dependiente entre los niños, trastorno que hasta la fecha había sido extremadamente raro en la infancia. Así mismo, los problemas del colesterol elevado ya no son exclusivos de las edades adultas, con su pésima repercusión en la futura hipercolesterolemia del individuo. El $50 \%$ de los niños obesos presentan lo que se denomina Asíndrome metabólico @ que constituye un factor de riesgo muy importante para padecer enfermedades cardiovasculares cuando lleguen a adultos. Se ha llegado a decir, incluso, que esta generación será una de las pocas que tendrá una esperanza de vida inferior a la de sus padres, ya que las enfermedades asociados al exceso de peso incrementan progresivamente el riesgo de mortalidad temprana cuanto mayor es el grado de sobrepeso.

Pues bien, este grave problema de nutrición está causado por unas graves disfunciones en los hábitos comportamentales 
alimenticios. Ahora bien, los hábitos alimenticios no sólo tienen que ver con el tipo de alimentos ingerido sino también con el cómo, cuándo y dónde se come (Amigo y Errasti, en prensa). Socialmente se ha instalado la creencia de que la prevención del sobrepeso pasa fundamentalmente por control del tipo de alimentos que se ingieren. Siendo cierto que el peso depende del tipo la alimentación que se consume, no se puede olvidar que lo que consumimos depende de otros hábitos que configuran el estilo de vida como por ejemplo las horas de sueño, el modo de afrontar la ansiedad o el tipo de ocio que disfrutamos (Christakis, Ebel, Rivara, y Zimmerman, 2004; Patrick, Norman, Calfas, Sallis, Zabinski, Rupp, y Cella, 2004; Spiegel K., Leproult, R., L'hermite-Baleriaux, M. et al, 2004; Spiegel, K., Tasali, E., Penev, P., Van Cauter E, 2004; Dennison, Erb y Jenkins, 2002). Es por ello que el objetivo de este estudio ha sido poner a prueba un modelo en virtud del cual se hipotetiza que dos hábitos propios del estilo de vida actual de la infancia, el ocio sedentario y dormir poco, se relacionan entre sí conformando un tándem obesogénico que facilita el aumento del sobrepeso y la obesidad infantil.

\section{MÉTODO}

\section{Participantes}

En esta investigación participaron 72 sujetos, 41 niñas y 31 niños, todos ellos estudiantes de 41 de Educacion Primaria en un colegio concertado de la ciudad de Oviedo y con una edad comprendida entre los 9 y los 10 años y medio. Para llevar a cabo la investigación en el colegio se solicitó a través del colegio la autorización a los padres de los alumnos para la participación de los niños en un estudio sobre hábitos alimenticios. Se garantizó la total confidencialidad de los datos y el anonimato de los participantes.

\section{Procedimiento}

La investigación se llevó a cabo a través de una entrevista individual con cada participante en un despacho del colegio. Al iniciar dicha entrevista el niño era pesado y medido sin calzado y con la ropa del 
uniforme para obtener su IMC. Después la investigadora se sentaba delante del niño ye le aplicaba el cuestionario que constaba de 14 cuestiones básicas y que indagaban sobre el consumo de horas de televisión, consola, ordenador, y el número de horas de sueño diarias. La entrevista duraba entre 20 y 25 minutos y cabe destacar el alto interés que ponían los niños en destacar los programas que veian y la valoración de los mismos. Para responder a las cuestiones sobre la horas de consumo de TV se les mostraba a los niños una guía de la televisión (TP) donde se podía precisar la duración real de los programas, de cara a obtener una mayor fiabilidad de las respuestas registradas. Así mismo se comprobaba la duración de los juegos con los que los niños decian jugar a la consola y ordenador.

\section{Material}

Para el pesaje de los niños se utilizó un báscula electrónica marca Firstline, modelo FPS4141. Se desarrolló un cuestionario sobre los hábitos de ocio sedentario y sueño (véase tabla 1).

\section{Análisis de resultados}

El análisis estadístico del modelo conceptual se realizó a través del path analysis o modelo de ecuaciones estructurales con el programa AMOS 6.0. Los análisis siguieron una lógica fundamentalmente confirmatoria. La evaluación del modelo se realiza en base a la significatividad del estadístico Chi-cuadrado, así como, a índices de bondad de ajuste GFI (Goodness of Fit Index), AGFI (Adjusted Goodness of Fit Index) y el RMSEA (Root Mean Square Error Aproximation).

\section{RESULTADOS}

El análisis descriptivo de los datos puso de manifiesto que la media semanal de consumo de TV en este muestra fue de 19.21 (DT =9,47) siendo, por lo tanto, la media diaria de 2,7 horas. Por lo que se refiere al juego con consola el numero medio de horas semanales fue de 3,4 $(D T=4,27)$ y con el ordenador $1,66(D T=2,22)$ La media diaria de horas 
Tabla 1. Cuestionario sobre hábitos de ocio sedentario y sueño

1.)A qué hora te levantas durante la semana para ir al colegio?

1.1. )A qué hora coges el autobús

2. )Dónde desayunas durante la semana

2.1. Cuándo desayunas ves la televisión?

2.2. Qué programas ves?

3. )Donde desayunas durante el fin del semana?

3.1.)Cuándo desayunas ves la televisión?

3.2. Qué programas ves?

4. )Dónde comes durante la semana?

4.1.) Cuándo comes ves la televisión?

4.2. Qué programas ves?

4.3. )Qué haces normalmente cuando sales del colegio al mediodia?

5. )Dónde meriendas durante la semana?

5.1. Cuándo meriendas ves la televisión?

5.2. Qué programas ves?

5.3. Qué haces normalmente cuando sales del colegio por la tarde?

6. )Dónde cenas durante la semana?

6.1. Cuándo cenas ves la televisión?

6.2. Qué programas ves?

7.) A qué hora te acuestas durante al semana normalmente?

8. )A qué hora te levantas el sábado y el domingo?

8.1.)Qué programas de televisión ves?

9. )A qué hora te acuestas el viemes, el sábado y el domingo?

9.1. Qué programas de televisión ves por la tarde y antes de acostarte?

10. ) Ves la televisión solo/a o con tus padres?

11. ) Tienes televisión en tu habitación?

12.) Tienes ordenador en tu habitación?

13. Cuántas horas al día juegas con el ordenador durante la semana?

13. 1.)A qué juegos sueles jugar con tu ordenador?

13.2.) Cuántas partidas juegas?

14. Tienes consola en tu habitación?

14.1. ) Cuántas horas al dia juegas con la consola durante la semana?

14.2. ) Cuántas horas al dia juegas con la consola durante el fin de semana?

14.3. )A qué juegos sueles jugar con la consola? 
de sueño fue 9,5 (DT=0,54) y la media del IMC fue de 18,64 (DT= 3,08 ). El numero total de participantes con sobrepeso fue 20 (el $28,8 \%$ de la muestra) y los casos de obesidad fueron 6 (el $8,6 \%$ de la muestra).

El path anaylisis reveló que el ajuste del modelo que se puso a prueba es bueno, ya que el chi-cuadrado no es significativo $(0,616)$ y los índices de ajuste $\mathrm{GFI}=0,996, \mathrm{AGFI}=0,978$ y RMSEA $=0,000$ son excelentes (ver gráfico 1). La matriz de correlaciones entre las variables aparece en la tabla 2.

Tabla 2. Matrlz de correlaclones de Pearson entre las horas de sueño, actividades de ocio sedentario y el IMC

\begin{tabular}{|l|l|l|l|l|l|}
\hline & $\begin{array}{l}\text { TOTAL } \\
\text { OCIO }\end{array}$ & $\begin{array}{l}\text { HORAS } \\
\text { TV }\end{array}$ & $\begin{array}{l}\text { HORAS } \\
\text { CONSOLA }\end{array}$ & $\begin{array}{l}\text { HORAS } \\
\text { ORDENADOR }\end{array}$ & IMC \\
\hline $\begin{array}{l}\text { HORAS DE } \\
\text { SUEÑO }\end{array}$ & $\begin{array}{l}-, 325 \\
\mathbf{P}=, 005\end{array}$ & $\begin{array}{l}-, 337 \\
\mathrm{P}=, 004\end{array}$ & $\begin{array}{l}-, 132 \\
\text { NS }\end{array}$ & $\begin{array}{l}-, 010 \\
\text { NS }\end{array}$ & $\begin{array}{l}-, 063 \\
\text { NS }\end{array}$ \\
\hline IMC & $\begin{array}{l}267 \\
\mathrm{P}=, 023\end{array}$ & $\begin{array}{l}, 238 \\
\mathrm{P}=, 044\end{array}$ & $\begin{array}{l}, 196 \\
\text { NS }\end{array}$ & $\begin{array}{l}, 006 \\
\text { NS }\end{array}$ & \\
\hline
\end{tabular}

En relación a las hipótesis que han configurado el modelo los resultados indican, en primer lugar y tal como era de esperar que el número total de horas de sueño mantiene una relación significativamente inversa con el ocio sedentario total (TV, ordenador y consola) ( $p<.05$ ) y el ocio sedentario predice a su vez un IMC elevado $(p<.05)$. Este modelo Aexplica una parte pequeña del IMC ( $7 \%$ de la varianza,) entre otras razones porque existe una relación bastante importante con la altura que no forma parte del interés del estudio.

\section{DISCUSIÓN}

Aunque, debido al tamaño de la muestra el objeto del estudio no fue cuantificar la prevalencia del sobrepeso y la obesidad infantil, si resulta llamativo que el porcentaje de sobrepeso encontrado de $28,8 \%$ refleja fielmente los datos que hoy se barajan para el conjunto de la población infantil española y que según los datos de la Agencia Española de Seguridad Alimentaria (2005) se situaría en el $26,3 \%$. 
Figura 1. Modelo de relaciones entre el sueño, total de ocio sedentario y el IMC. Los pesos significativos a un $5 \%$ figuran en rojo. Las variables son Sexo, Sue (horas de sueño), Total (horas de ocio empleadas en la TV, consola y ordenador) e IMC

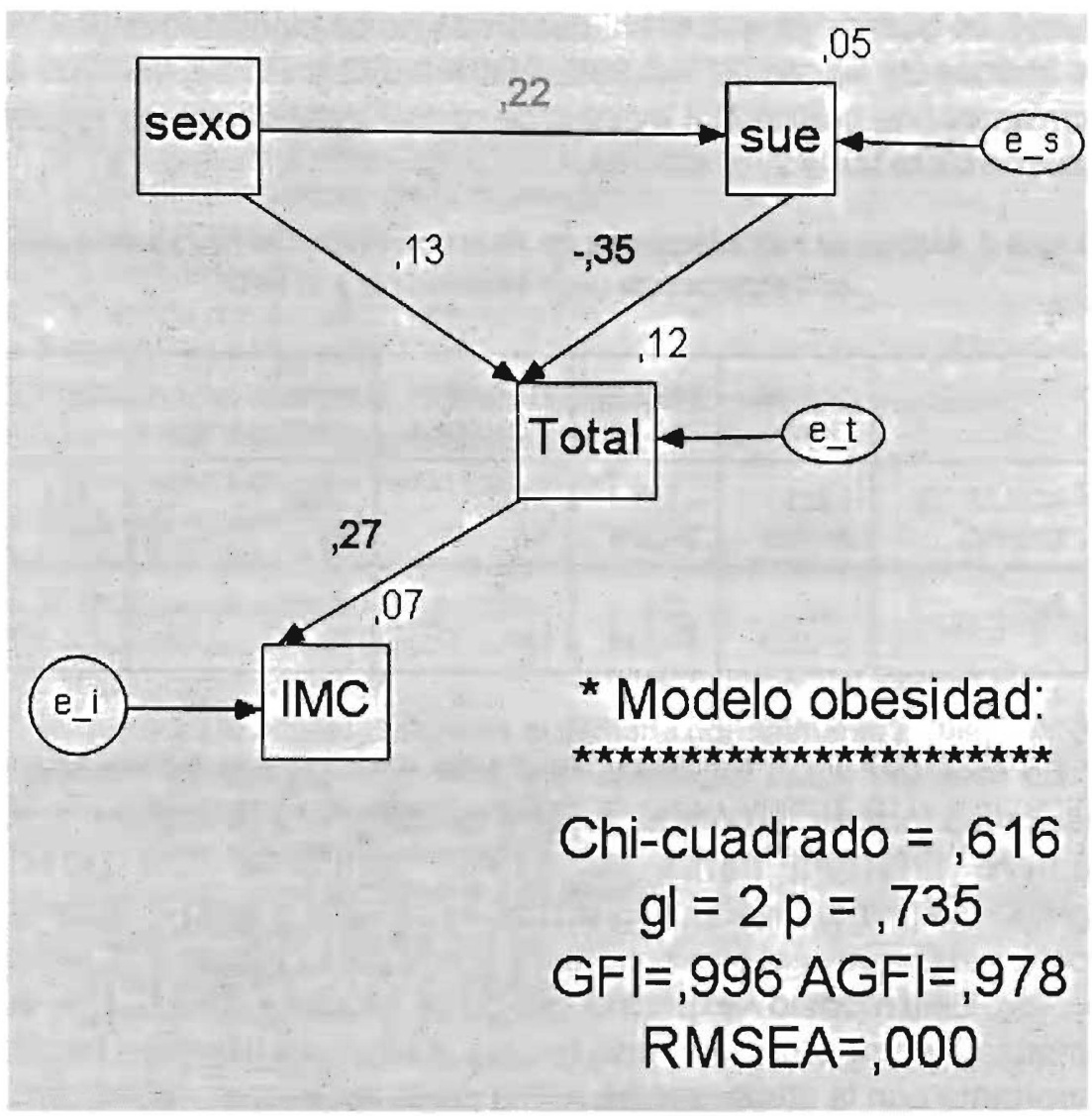

Por lo que respecta al objetivo de estudio de analizar como el ocio sedentario y dormir poco, se relacionan entre si conformando un tándem obesogénico que facilita el aumento del sobrepeso y la obesidad infantil, los datos parecen avalar esta asociación. Recientes investigaciones han señalado que existe una relación inversa entre el número de horas de sueño y el IMC de tal forma que los niños que duermen menos horas muestran un IMC mayor que los niños que duermen más 
(Chaput, Brunet y Tremblay, 2006; Von Kries, Toschke, Wurmser, Sauerwald y Koletzko, 2002). El modelo de ecuaciones estructurales que hemos realizado permite configurar una relación compleja entre la falta de sueño, el ocio sedentario y el IMC. Las horas que los niños quitan al sueño parecen emplearlas en alguna forma de ocio sedentario y ese mayor número de horas de entretenimiento sedentario es el que llevaría al aumento del IMC. En concreto, hemos observado que la falta de sueño correlaciona inversamente con el número total de horas de ocio sedentario que los niños emplean fundamentalmente viendo y jugando con la TV, la consola y el ordenador. Lo que quiere decir muy probablemente que los niños que duermen menos invierten ese tiempo, que deberían estar durmiendo, jugando de un modo sedentario. Así, en este trabajo se ha evidenciado una relación indirecta entre las horas de sueño y el IMC que estaría mediada por el número total de horas dedicadas al ocio sedentario.

En este sentido, el modelo pone en evidencia que el número de horas de ocio sedentario predice, de un modo directo, un IMC elevado. Este hecho ha sido observado sistemáticamente desde medidados de los años 80 hasta la actualidad (Dietz, y Gortmaker, 1985; Christakis, Ebel, Rivara, y Zimmerman, 2004). Esta forma de ocio reduce notablemente el gasto energético del organismo. No se debe olvidar que delante de las TV el número de calorías que se consumen son prácticamente las mismas que si el niño estuviera durmiendo. Es por ello limitar el número de horas de TV y las otras formas de ocio sedentanio parece constituir una herramienta fundamental para la prevención de la obesidad infantil (Robinson, 1999; Gortmaker, Peterson, Wiecha, Sobol, Dixit, Fox, y Laird, 1999). En concreto, parecería adecuado recuperar el hábito de marchar a una hora predeterminada a la cama (en función de la hora a la que el niño tenga que levantarse) y no en función de programa de televisión que se este viendo.

En particular, resulta de especial interés la relación que se ha observado, a pesar del pequeño tamaño de la muestra utilizada, entre la falta de sueño y el IMC mediada por el ocio sedentario. Una relación que la investigación futura no debería obviar. La relación entre la falta del sueño y el incremento podría ser explicada por los desajustes hormonales, en concreto, de la grelina y leptina. La leptina, es la responsable de avisar al cerebro del estado de saciedad, y la grelina, 
estimula la sensación de apetito. Los resultados de algunos estudios indican que la falta de sueño provoca en los jóvenes un cambio en estas hormonas, lo cual les hacia tener más apetito y les provocaba más dificultades para sentirse saciados. Además, el deseo de alimentos que se incrementa más marcadamente es específicamente el de dulces y galletas, patatas fritas o comidas saladas (Spiegel, Leproult, L'hermite-Baleriaux, Copinschi, Penev, Van Cauter, 2004; Spiegel, K., Tasali, E., Penev P. y Van Cauter, E. (2004).

Todos estos datos indican, en definitiva que, para entender el sobrepeso infantil, que en la actualidad sigue incrementándose, sería necesario desarrollar un modelo que no sólo contuviese estas dos variables sino que, además del tipo de alimentación que los niños consumen, se añadiese otros hábitos tales como comer solos o comer para calmar el aburrimiento. De esta manera se dispondria de una herramienta útil que permitiría definir todos aquellos hábitos que conforman la constelación de vectores responsables del sobrepeso infantil y establecer el marco adecuado para su prevención.

\section{REFERENCIAS BIBLIOGRÁFICAS}

Agencia Española de Seguridad Alimentaria (2005). Estrategia NAOS. Madrid: Ministerio de Sanidad y Consumo.

Amigo, I. y Errasti, J.M. (en prensa). Los nueve habitos que causan la obesidad infantil. Bilbao: DDB.

Chaput, J.P., Brunet, M.y Tremblay, A.(2006). Relationship between short sleeping hours and childhood overweight/obesity: results from the AQuebec en Forme@ Project. International Journal of Obesity. Advanced online publication.

Christakis, D.A., Ebel, B.E., Rivara, F.P., y Zimmerman, F.J. (2004). Television, video, and computer game usage in children under 11 years of age. The Journal of Pediatrics, 145, 652-656.

Dennison, B.A., Erb, T.A., y Jenkins, P.L. (2002). Television viewing and television in bedroom associated with overweight risk among low-income preschol children. Pediatrics, 109, 1028-1035.

Dietz, W.H., y Gortmaker, S.L. (1985). Do we fatten our children at the television set? Obesity and television viewing in children and adolescents. Pediatrics, 75, 807-812. 
Flegal, K.M. (2005) Epidemiologic aspects of overweight and obesity in the United States. Physiology and Behavior, 2005 Oct 18; Garcés, C., Gutierrez, J., Benavenente, M. (2005) Obesity in Spanish schoolchildren relationship with profile and insulin resistence. Obes Res. 13:959-63.

Gortmaker, S.L., Peterson, K.E., Wiecha, J., Sobol, A.M., Dixit, S., Fox, M.K., y Laird, N. (1999). Reducing obesity via a school-based interdisciplinary intervention among youth (Planet Health). Arch Pediatr Adolesc Med, 153, 409-418.

Patrick, K., Norman, GJ., Calfas, K.J., Sallis, J.F., Zabinski, M.F., Rupp, J., y Cella, J. (2004). Diet, physical activity, and sedentary behaviors as risk factors for overweight in adolescence. Archives of Pediatric \& Adolescence Medicine, 158, 385-90.

Robinson, T.N. (1999). Reducing children's television viewing to prevent obesity: a randomized controlled trial. Journal of American Medical Association, 282, 1561-1567.

Spiegel, K., Tasali, E., Penev P. y Van Cauter, E. (2004). Brief communication: Sleep curtailment in healthy young men is associated with decreased leptin levels, elevated ghrelin levels, and increased hunger and appetite. Annals of Internal Medicine, 141, 885886.

Spiegel K., Leproult, R., L'hermite-Baleriaux, M., Copinschi, G, Penev, P.D., Van Cauter, E. (2004). Leptin levels are dependent on sleep duration: relationships with sympathovagal balance, carbohydrate regulation, cortisol, and thyrotropin. The Journal of Clinical Endocrinology \& Metabolism, 89, 5762-5771.

Von Kries, Toschke, A., Wurmser, H., Sauerwald, T., Koletzko, B. (2002).

Reduced risk for overweight and obesity in 5 and 6 year old children by duration of sleep. A cross sectional study. International Journal of Obesity and Rlated Metabolic Disorders,26, 710-716. 